Cinémas

Revue d'études cinématographiques

Journal of Film Studies

\title{
L'institution du film de fiction aux États-Unis : le point de vue sémio-pragmatique
}

\section{Pierre Barrette}

Volume 7, numéro 3, printemps 1997

Cinéma québécois et États-Unis

URI : https://id.erudit.org/iderudit/1000947ar

DOI : https://doi.org/10.7202/1000947ar

Aller au sommaire du numéro

Éditeur(s)

Cinémas

ISSN

1181-6945 (imprimé)

1705-6500 (numérique)

Découvrir la revue

Citer cet article

Barrette, P. (1997). L'institution du film de fiction aux États-Unis : le point de vue sémio-pragmatique. Cinémas, 7(3), 13-29. https://doi.org/10.7202/1000947ar
Résumé de l'article

Après une brève synthèse des diverses approches disciplinaires de la notion de contexte mettant en relief la spécificité du modèle sémio-pragmatique, cet article essaie de répondre aux questions suivantes : comment l'organisation des sphères de production pèse-t-elle sur le contenu lui-même des films? quel spectateur est sous-entendu par ce modèle? quel sens y prend la notion d'auteur? étant donné cette situation générale, sur quelles bases peut s'effectuer la réception du cinéma étranger? 


\title{
L'institution du film de fiction aux États-Unis : le point de vue sémio-pragmatique
}

\section{Pierre Barrette}

\begin{abstract}
RÉSUMÉ
Après une brève synthèse des diverses approches disciplinaires de la notion de contexte mettant en relief la spécificité du modèle sémio-pragmatique, cet article essaie de répondre aux questions suivantes: comment l'organisation des sphères de production pèse-t-elle sur le contenu lui-même des films? quel spectateur est sous-entendu par ce modèle? quel sens y prend la notion d'auteur? étant donné cette situation générale, sur quelles bases peut s'effectuer la réception du cinéma étranger?
\end{abstract}

\section{ABSTRACT}

After a brief summary of the various disciplinary approaches to the notion of context, focusing specifically on the semio-pragmatic model, this article attempts to answer these questions: How does the organization of film production influence the content of films? What kind of spectator is implicit in this model? What significance is accorded to the notion of an author? Given this overall situation, on what basis will the reception of foreign films be realized?

C'est presque devenu un lieu commun aujourd'hui, dans le champ de la théorie du cinéma, que de postuler l'importance du pôle de la destination (qu'on nomme celui-ci spectateur, lecteur, récepteur, interlocuteur, etc.) dans l'analyse des différents processus de production de sens. En effet, on a bien vu comment ce 
qu'on a qualifié de sémiologie de première génération, informée par le structuralisme et bien assise sur le postulat de l'autonomie signifiante des textes, a vite été confrontée à toutes sortes de difficultés, dont la moindre n'est pas la question du contexte de production et de réception des œuvres tel qu'il exerce une influence non négligeable dans l'attribution du sens (Eco, 1985). C'est ce postulat immanentiste qui est remis en question par la sémiologie de deuxième génération, ainsi que par une série d'autres disciplines connexes qui s'intéressent au fait filmique et qui ont en commun la volonté de redonner au destinataire la place qui lui revient.

Une bonne part des développements récents dans la théorie du cinéma paraît être le résultat direct de cette sensibilité plus grande des chercheurs à ce qu'il semble convenu d'appeler le "contexte" des films. Contexte de production et de réception, contexte social et culturel, contexte cinématographique et contexte historique: c'est comme si le champ de l'analyse du cinéma et des films s'était ouvert tout à coup à la pluridisciplinarité, en réaction probablement à l'immanentisme des modèles interprétatifs en vogue dans les années soixante-dix et au début des années quatre-vingt. C'est en ce sens qu'il faut comprendre, croyons-nous, l'apport de plus en plus important de disciplines comme l'histoire de la réception, de la psychologie cognitive ou de la linguistique pragmatique au domaine des études cinématographiques, mais aussi l'émergence de problématiques nouvelles, comme celles qui animent le présent numéro de la revue Cinémas.

Dans cette perspective, le présent article se donne un double objectif: 1) proposer une brève synthèse de diverses approches disciplinaires de la notion de contexte, ce qui nous permettra de mettre en relief la spécificité du modèle sémio-pragmatique; 2) dès lors, soumettre de nouveaux éléments de réponse à certaines questions fréquemment soulevées dans d'autres cadres: comment l'organisation des sphères de production pèse-t-elle sur le contenu lui-même des films? quel spectateur est sousentendu par ce modèle? quel sens y prend la notion d'auteur? étant donné cette situation générale, sur quelles bases peut s'effectuer la réception du cinéma étranger? 


\section{De l'énonciation à la réception}

Il apparaît possible et même souhaitable d'envisager la question du contexte par rapport au degré de concrétude qu'on accorde à la figure du spectateur. On se trouve de cette façon placé devant un spectre de constructions spectatorielles dont les pôles correspondent d'une part au spectateur énoncé par le film (ce qu'il est convenu d'appeler l'étude de l'énonciation), et d'autre part au spectateur empirique (ce pôle correspondant à l'étude de la réception concrète des films). Entre ces deux positions extrêmes, une infinité potentielle de figures intermédiaires existe bien sûr, mais pour les besoins de la présente synthèse, nous allons nous concentrer sur cette importante polarisation qui agit dans le champ des études cinématographiques comme un jalon clair des options qui s'offrent à l'analyste. Voyons cela de plus près.

Du petit texte "Notes sur deux voyeurismes" (Metz, 1984) naîtra ce qu'on peut sans contredit considérer comme la conception contemporaine la plus influente et la plus largement commentée des rapports entre film et spectateur. Cette conception, qu'on qualifiera de métapsychologique et textuelle, part de la distinction établie par Benveniste (1966) entre l'histoire (définie comme un texte dans lequel toutes les marques de communication entre le destinateur et le destinataire sont effacées) et le discours (dans lequel, au contraire, ces marques sont présentes), couplée à la distinction établie par la psychanalyse freudienne entre voyeurisme et exhibitionnisme. On cherche ainsi à fournir une explication métapsychologique à l'absence de marques énonciatives dans la majorité des films de fiction. Selon Metz, le film narratif-représentatif "classique" (entendre: celui qui se constitue sur le modèle hollywoodien) représente un discours qui a effacé les signes de sa destination pour se déguiser en histoire; une fois débarrassé de ces marques qui le situeraient dans le circuit d'un échange (de type économique), le film se donne à voir comme s'il venait de nulle part et n'était destiné à personne, permettant par là au spectateur de jouir du plaisir voyeuriste sans être "dérangé".

La figure spectatorielle dont il est question dans cette sémiologie de l'énonciation est un spectateur "positionné" par le 
dispositif cinématographique. En accord avec la définition qu'en donne Metz, il ne s'agit pas de l'individu en chair et en os qui se rend au cinéma, mais bien la part de lui-même qui y va. En fait, c'est le sujet idéologique tel que l'institution du cinéma de fiction le conçoit, et la place, en creux, que les films lui réservent.

Linstitution du cinéma prescrit un spectateur immobile et silencieux, un spectateur "dérobé", constamment en état de sous-motricité et de sur-perception, un spectateur aliéné et heureux, acrobatiquement raccroché à luimême par le fil invisible de la vue, un spectateur qui ne se rattrape comme sujet qu'au dernier moment, par une identification paradoxale à sa personne propre, exténuée dans le regard pur (Metz, p. 119).

Cette manière de concevoir les rapports qui se tissent entre un film et son spectateur en termes de "rêverie éveillée" n'est pas sans rappeler la célèbre analogie utilisée par Jean-Louis Baudry (auquel Metz se réfere par ailleurs ouvertement) pour décrire ce même dispositif de base. En effet, ce dernier évoque la métaphore de la caverne de Platon pour illustrer la situation de manipulation inconsciente dont est victime le spectateur: "Projection et réflexion se produisent dans un espace clos, et ceux qui y demeurent se trouvent qu'ils le sachent ou non (mais ils ne le savent pas) enchaînés, capturés ou captés" (Baudry, p. 23). On peut aisément qualifier d'idéal et de textuel ce mode de construction de la figure spectatorielle, et ce qu'on étudie ici, c'est bien plus, en accord avec la tradition littéraire du lecteur idéal ou compétent, une visée du film, une sorte de point aveugle tout à la fois produit par le texte et comme projeté hors de lui, une condition de lisibilité, dont les termes sont largement a-historiques et finalement assez indépendants de tout acte de lecture ou de spectature, de tout contexte.

En tant qu'il est lisible dans le texte sous la forme d'une destination, on est en droit de se demander, comme certains déjà l'ont fait de façon convaincante, si un tel spectateur n'est pas une sorte de résurgence en forme de compromis des "structures signifiantes " et autres codes propres aux analyses immanentistes, certes pas très au goût du jour, mais tenaces quand il s'agit de ne pas sacrifier à l'autel du relativisme l'idée selon laquelle certaines 
lectures sont plus pertinentes que d'autres. Il apparaît en ce sens que toutes les analyses qui fondent leur conception du spectateur sur une structure énonciative propre au texte, comme par exemple la narratologie (Jost, 1988), si elles ont bien leur valeur en tant qu'outil analytique, semblent assez peu pertinentes quand vient le temps de rendre compte du contexte effectif d'un film et de son poids sur la lecture.

A l'autre bout du spectre, les tenants de l'approche dite «de réception" s'intéressent au spectateur empirique. Pour Janet Staiger (1987 et 1992), par exemple, le spectateur idéal de la sémiologie d'inspiration linguistique ne correspond en fait qu'à une position spectatorielle possible parmi celles disponibles à un moment donné; en faire le pivot de l'analyse revient pour le chercheur à poser son interprétation comme la seule valable. Ce qu'il faut voir, par ailleurs, c'est que la position inverse, qui consiste à admettre la légitimité de toutes les lectures, joue le jeu de l'idéologie bourgeoise selon laquelle l'individu est "libre" de ses interprétations. L'une et l'autre position, pour la chercheure états-unienne, rendent nécessairement compte de manière incomplète des processus de réception et d'interprétation des films, parce que trop centrée sur le texte pour l'une, et trop occupée par le récepteur pour l'autre.

Le contexte dans lequel se produit l'acte de communication est primordial pour Staiger, qui définit son approche par sa capacité à relativiser le centralisme propre aux analyses textuelles :

As I envision its field of research in cinema, its central questions would be: (1) What have been the reception of cinema / genres / specific films / movements and (2) what explains these receptions? [...] For reception studies ought be neither a theory nor a textual procedure; [it should be] a critical tool or intervening practice which dislodges attempts at centering and stabilizing theories and knowledges as privileged or true or hierarchical (1989, p. 354).

Lobjet d'analyse n'est plus le texte filmique proprement dit, mais bien les réactions, opinions, interprétations qu'il suscite ou a suscitées à un moment donné. 'C'est dire tout ce qui sépare cette approche des théories dites de la réception en littérature 
qui restent essentiellement textuelles dans leur méthode. Dans le cadre proposé par Staiger, le spectateur n'est pas positionné ou déduit, mais historiquement attesté, et ce qui est analysé à travers lui, c'est non pas la validité, mais la possibilité même d'une interprétation qui trouve nécessairement, dans les conditions historiques synchroniques de sa production, une raison transcendant le simple libre arbitre individuel.

Dans ce contexte, aucune interprétation suscitée par un texte n'est jugée impertinente, erronée, non plus que meilleure qu'une autre. Quelle qu'elle soit, elle est susceptible de jeter un éclairage sur la position tenue par le locuteur dans le filet social, et par là, de mieux rendre compte des enjeux politiques de la communication; à ce titre, elle est toujours significative. Le spectateur n'est jamais entièrement libre de produire la signification qu'il veut : certaines contraintes sont textuelles, bien sûr, mais bien d'autres ont trait à la compétence particulière de l'individu. Et cette compétence, contrairement à certains chercheurs qui la placent dans le texte, comme une sorte de condition de la lecture, Staiger la trouve dans l'interprétation, comme la résultante du positionnement social et institutionnel de son producteur.

D'où l'importance de ce qu'elle nomme les "ideolects of reception", ces formations sociales plus ou moins bien délimitées et qui peuvent servir de base à la compréhension d'une saisie, en termes politiques, de la variation de la réception:

A comparative approach could consider «ideolects" of reception across nations (questions of social formations at large scale); cultural groups (question of perhaps reified, but socially real and effective, classifications such as race, ethnicity, and class); sexual differences (questions of gender, socialized masculinity and feminity, sexual orientation); political sympathies and beliefs; and popular versus academic procedures (1989, p. 364).

Du point vue méthodologique, un tel programme doit permettre de fournir un ensemble de données pertinentes pour l'analyse, données qui correspondent à la réception effective d'individus historiques. Comme cela a toujours été le cas pour les études de types empiriques s'intéressant à la "spectature", c'est la localisation et la collecte proprement dite de ces données 
qui soulèvent de nombreux problèmes. Il est bien sûr possible de mener des entrevues à la sortie des cinémas; mais outre le fait qu'il est loin d'être certain que la réponse à une entrevue soit à même de restituer ce que fut la réception du film, cette méthode ne fournit que des données contemporaines de la recherche. La solution qui semble s'imposer tout naturellement, et qui n'a pas tardé à devenir la norme en matière d'analyse concrète de la réception historique des films, est l'utilisation des critiques, commentaires, reviews, analyses savantes, résumés, etc., qui ont suivi et dans certains cas continuent à suivre la sortie d'un film (voir en ce sens Müller, 1992).

As a text it is readable and available for analysis as any other texts via the methods used by the critics to study literary texts. In other words, if we can't read a review symptomatically, then we might as well give up the notion of reading any text. Furthermore, if a review is placed into a comparative relation with other reviews generated from analogous circumstances, certain similarities and differences begin to be observable and potentially pertinent. In addition, accross time, other caracteristics become observable (Staiger, p. 362).

Indépendamment des tenants et des aboutissants d'une telle approche que nous considérons, par ailleurs, nécessaire dans le champ des études cinématographiques comme " complément " à l'analyse textuelle, il nous semble que les choix méthodologiques qui l'ont guidée jusqu'ici ne lui permettent pas de remplir adéquatement son mandat. En effet, même si on laisse de côté le fait que les textes critiques et les autres commentaires de films se constituent de bases génériques (ce qui, comme l'a bien vu Staiger elle-même, modifie profondément l'expression même de ce qu'a pu être la réception), il faut bien se rendre compte qu'ils sont écrits par des professionnels du journalisme, de l'Académie ou d'une autre institution qui, du point de vue néomarxiste adopté par Staiger, ont toutes les chances de parler à peu de chose près le même "idiolect". Il est bien possible qu'une telle contrainte soit inévitable; mais dès lors, l'analyse de la réception pourrait fort bien ressembler à l'analyse des différents discours institutionnalisés sur le cinéma. Une telle démarche n'est certes 
pas inutile, mais elle s'avère assez éloignée du projet original qui consiste dans l'étude du spectre des interprétations possibles d'un film à un moment donné de l'histoire.

Avec les approches centrées sur la réception, c'est à un renversement complet des mécanismes de production de la signification auquel on assiste. Pour nous, les critiques adressées à la sémiotique et aux analyses textuelles qui construisent un spectateur de type idéal sont justes, dans la mesure où ces méthodes placent trop l'emphase sur le pouvoir contraignant du texte et rendent par là trop peu compte du poids idéologique et circonstanciel qui sous-tend leur propre position de lecture. Les études sur la réception nous montrent l'importance de considérer les enjeux sociaux et politiques de la communication, et nous forcent à conserver une attitude critique et distanciée vis-à-vis de nos propres lectures de textes; c'est particulièrement vrai de la lecture de type universitaire qui est la nôtre et qui traditionnellement n'a pas toujours mis de l'avant ce type d'attitude réflexive. Mais en partant des interprétations historiques et effectives des textes pour remonter vers leurs origines (idéologiques ou autres), ce qu'on semble oublier c'est que, symétrique au contexte de lecture, pèse également sur le sens du texte un contexte de production; car s'il existe quelque chose comme des communautés interprétatives, localisables historiquement, socialement et géographiquement, il existe également quelque chose comme une "communauté d'auteurs" qui contribue, elle aussi, au façonnement du sens dans les œuvres. Si, comme l'affirme Staiger, toutes les interprétations ne sont pas possibles, il faut bien reconnaitre que tous les textes non plus ne sont pas "produisibles». Le contexte pèse aussi fortement sur les auteurs que sur les lecteurs-spectateurs et, conséquemment, affirmer que toutes les lectures produites historiquement sont explicables et donc pertinentes revient à nier l'existence d'une force auctoriale (qu'on définira comme l'artiste, le groupe, la société, peu importe) à l'œuvre dans le film et qui possède ses visées propres, elles aussi explicables.

Entre ces deux manières antinomiques de construire la figure spectatorielle, correspondant à des conceptions opposées du contexte et de son rôle, on trouve bien sûr des positions intermé- 
diaires: les travaux de David Bordwell (1985 et 1989) pour intégrer dans le cadre des analyses filmiques des considérations issues de la psychologie cognitive, par exemple, ou encore les tentatives les plus récentes de François Jost (1992) pour ouvrir le concept d'énonciation sur une véritable dynamique communicationnelle, en font foi. Ces approches restent toutefois assez timides dans leur définition d'un cadre permettant de tenir compte du contexte des films. En réalité, seule l'approche sémio-pragmatique constitue de notre point de vue une tentative sérieuse d'intégration du contexte dans le cadre de l'analyse textuelle.

\section{La sémio-pragmatique du cinéma : \\ contexte, institution, contrat}

Le théoricien du cinéma Roger Odin (1983a et b, 1988 et 1989) a été l'un des premiers à proposer le renouvellement du cadre sémiotique par la pragmatique, et certainement celui qui, depuis lors, poursuit avec le plus de consistance cet objectif. Le point de départ et catalyseur des recherches du chercheur français trouve son origine dans une insatisfaction vis-à-vis de la démarche analytique: "Prenons l'exemple des analyses textuelles: non seulement, en général, elles ne disent rien des déterminations externes qu'elles présupposent, ni du type de spectateurs qu'elles mettent en œuvre, mais rares sont celles mêmes qui témoignent qu'elles ont conscience du problème" (1989, p. 81). Pour lui, la raison en est assez claire : parce que traditionnellement, la théorie du cinéma dans son ensemble s'est presque exclusivement intéressée au cinéma dominant, le cinéma dit "narratif-représentatif", on a cessé d'éprouver le besoin de se questionner sur les déterminations institutionnelles qui lui président. Conséquemment, de nombreuses questions ayant une incidence déterminante sur le processus de signification sont prises comme allant d'elles-mêmes: qu'est-ce que la fiction? pourquoi est-ce le mode dominant au cinéma? qu'est-ce qui caractérise le spectateur de fiction? quelle est la part de déterminations institutionnelles qui entre dans la compréhension d'un film? Toutes ces interrogations, Odin les formule en priorité, à côté d'autres questions plus traditionnelles qui ne seront abordées qu'une fois résolu le problème du cadre institutionnel dans lequel se situe un film donné. 
Cette nécessité de toujours commencer par comprendre et analyser les déterminations externes du film avant d'en analyser le contenu et la structure a amené Odin à s'intéresser au champ cinématographique dans son ensemble, contrairement à la plupart des sémiologues qui se penchent sur la dimension filmique des œuvres sans grand égard pour ce qui relève de considérations sur le contexte. Ainsi, pour le chercheur, la signification de tel ou tel élément dans un film ne saurait être appréhendée sans référence à la catégorie d'appartenance du film en question : par exemple, dans une œuvre participant de la catégorie du film d'essai ou du film d'art, le regard à la caméra apparaît comme quelque chose de relativement fréquent et d'assez anodin, dont la signification est codée et comprise par le spectateur entraîné à la lecture de ces films; le même regard à la caméra dans une comédie musicale hollywoodienne des années trente prend une tout autre signification; dans un documentaire, ce même élément prendra un troisième sens, etc. On comprend bien qu'il est impossible dans un tel contexte de croire à un sens immanent des textes ou des films. La notion d'institution apparait en ce sens comme un troisième terme permettant de rendre compte d'un espace de manœuvre à l'intérieur duquel les protagonistes de la situation de communication jouissent d'une liberté limitée.

Un film qui n'a pas été conçu dans l'espace de la réalisation comme un film de fiction peut tout de même être vu sur le mode fictionnel dans l'espace de la lecture pour peu que le blocage ne fonctionne pas à tous les niveaux. Il existe donc une lecture fictionnalisante relativement indépendante du film lui-même (Odin, 1988, p. 129).

Dire comme les sémiologues de la première génération qu'un film signifie telle ou telle chose en fonction de tel ou tel procès de signification, ou au contraire avancer comme les tenants d'une approche intéressée par la réception qu'un film signifie ce que les spectateurs y voient, constituent deux manières diamétralement opposées de couper le film du contexte de son occurrence. Autrement dit, pour nous, il est tout aussi absurde de postuler que le sens est immanent au texte que de poser a priori que toutes les lectures attestées sont légitimes. La production et 
la réception d'artefacts culturels ne s'effectuent pas dans leur vase clos respectif, mais dans un vase commun qu'on nomme l'institution. Conséquemment, ce que propose la sémio-pragmatique, c'est d'approcher les films selon une méthode qui soit prioritairement textuelle (c'est aux films qu'on s'intéresse; les réactions, critiques, lectures qu'ils suscitent sont là comme symptômes, jamais comme fins de la recherche), tout en ouvrant sur une conception des œuvres comme objets communicationnels.

Nous ne pouvons pas pour autant suivre jusqu'au bout les prolongements méthodologiques que donne Odin à ces principes fondamentaux, pour leur part incontournables. En effet, fidèle au postulat de base de la sémiologie plus traditionnelle, l'auteur rejette toute concession à l'empirisme et postule que la sémio-pragmatique doit s'intéresser aux conditions de possibilités générales de la signification, ce qu'il nomme "[...] les conditions de possibilité du code" (1989, p. 90). Nous arrivons mal à comprendre comment Odin arrive à distinguer ce qui relève d'une part des modes de production fondamentaux, intériorisés par les sujets, et d'autre part de ce qui relève de leur manifestations. Peut-on imaginer en effet que l'idée de fiction cinématographique existe en dehors des occurrences historiques qui l'ont générée et qui continuent à en modifier les contours? Doit-on établir les grandes fonctions remplies par le documentaire avant même de se pencher sur ses occurrences historiques? Et ne fausse-t-on pas de la sorte les résultats qu'on peut espérer tirer de l'analyse?

Un court détour du côté de la théorie de l'institution littéraire nous permettra de concrétiser notre définition de l'institution tout en gardant en perspective le nécessaire équilibre avec l'analyse textuelle. Rapprochant l'institution littéraire des autres institutions fonctionnant dans l'espace social, et tentant de rendre compte des modes spécifiques de leur action, Jacques Dubois (1986) définit celles-ci comme des "[...] ensembles de normes s'appliquant à un domaine d'activités particulier et définissant une légitimité dans une charte ou un code" (p. 31). En ce sens, ce qui nous intéresse au premier chef, ce sont les rapports de déterminations qui existent entre la structure de l'institution et la 
structure textuelle; autrement dit, comment l'organisation géographico-historique spécifique de production et de réception travaillent l'ensemble des faits discursifs. Les textes que l'on produit, la manière avec laquelle on les fait, le sens qu'on leur prête, toutes ces questions relèvent selon nous, du moins en partie, de déterminations institutionnelles qu'on nommera avec Benoît Melançon (1986) les "faits d'appareils». C'est que les institutions, contrairement à la manière dont on a tendance à les traiter dans le champ des études cinématographiques en particulier, relèvent aussi de structures concrètes (instances de production et de légitimation, instances critiques, réseaux de diffusion, publics, etc.) dont la nature et l'organisation spécifiques pèsent sur les aspects textuels à proprement parler.

D'autre part, on appellera "faits de discours" ces niveaux plus directement rapportables au mode d'organisation interne, déterminés selon un rapport variable par les faits d'appareils. Ceux-ci varient bien sûr d'un lieu, d'une époque et d'une institution à l'autre; en fait, c'est toujours à l'analyste de fixer les particularités de son objet en fonction de sa ou de ses questions de recherche. La notion de "contrat de communication" est celle qui recouvre le mieux l'ensemble des opérations signifiantes, telles qu'un film les prescrit à son spectateur, en fonction d'un cadre institutionnel donné. En effet, comme le mentionnent Casetti et Odin, l'institution apparaît justement comme " [...] une structure régissant dans son espace propre le recours à tel $(s)$ ou tel(s) contrat de communication" (Casetti et Odin, p. 9).

Comme on le verra dans la dernière partie de cet article, prendre en considération la double structure institutionnelle du cinéma, ses aspects hors et intra-textuels, ouvre à l'analyse des perspectives renouvelées, qui respectent l'exigence à maintes fois formulée de saisir les phénomènes filmiques à l'intérieur des circonstances de leur production-réception.

\section{L'institution du film de fiction aux États-Unis : quelques problèmes}

Nous voulons, pour terminer, donner quelques exemples de problèmes bien contemporains souvent soulevés par la théorie ou par une certaine presse spécialisée et qui trouvent dans 
l'éclairage particulier de la sémio-pragmatique des éléments de solutions pertinents. Bien sûr, nous n'entendons pas traiter de ces problèmes de manière exhaustive; il s'agit plutôt d'indications et de pistes de réflexions pour un travail futur.

Le premier problème concerne ce que la théorie de l'institution nomme les sphères de production. Jacques Dubois a bien montré, pour le domaine de la littérature en France, l'existence de deux champs, séparés mais complémentaires, auxquels correspondent des finalités et des modes de productions différents. Cette distinction est d'autant plus importante que c'est elle qui donne son relief particulier à l'ensemble de l'institution de la littérature, établissant en réalité un partage souvent arbitraire entre le légitime et l'illégitime. Selon ce partage, on trouve d'une part le champ de grande production, et d'autre part, le champ de production restreinte; d'un côté la littérature de masse et, de l'autre, la littérature lettrée, chacune de ces sphères définissant des pratiques d'édition, de production et d'écriture qui lui sont propres. Bien sûr, la limite entre les deux sphères peut être floue, des croisements et des variations sont inévitables, mais dans l'ensemble, voilà deux territoires assez clairement séparés où, on s'en doute, c'est le champ de production restreinte qui a de son côté la "légitimité ", qui fait figure d'institution dominante dans l'ensemble du tableau littéraire français, le champ de grande production et son produit étant assimilé à une sorte de sous-culture marchande.

Quand on compare cette situation à celle de l'institution cinématographique des États-Unis, ne serait-ce que superficiellement, des différences fort éclairantes apparaissent. En effet, contrairement à ce qui se passe en littérature, et pour des raisons qui tiennent autant au statut du médium qu’à ses contingences, la sphère de production restreinte est en position d'infériorité par rapport à Hollywood. Les critères de rentabilité imposés par le marché font en sorte qu'il est de plus en plus difficile pour les auteurs de travailler à une ouvre personnelle, s'éloignant des standards de la production courante. Dire que cette situation est nouvelle serait aberrant: on connaît trop bien les difficultés qu'a pu connaître un Orson Welles, des années quarante aux années soixante-dix, par exemple, pour croire que cet ostracisme nous 
soit contemporain. Il est clair toutefois que la logique qui règne actuellement et ce, depuis bientôt une vingtaine d'années, celle des blockbuster (la production d'un petit nombre de films, extrêmement coûteux, accompagnée d'une campagne de marketing monstre, dont le succès au box office est presque assuré) contribue pour beaucoup à l'effondrement du poids relatif des petites productions dans l'ensemble du budget des studios, Majors et Indépendants. Comme le dit bien cet analyste de la situation contemporaine dans le cinéma des États-Unis :

As those investments grow astronomically, they do much more than simply increase the size of the stakes that once were invested in classical film stars of the forties or $3 \mathrm{D}$ technology in the fifties. They alter the very nature of film product by forcing a massive alteration of the conception of an audience [...] Those investments and technology must aim to "undifferentiate" the desire of different audiences, usually by emphasising the importance of that investment "in and of itself " (Corrigan, p. 20-21).

Autrement dit, le champ de grande production (caractérisé par une structure industrielle lourde, des investissements faramineux et une recherche du rendement immédiat), parce qu'il oblige à une capitalisation maximale d'un minimum de films, se trouve devant la nécessité de proposer des films susceptibles de plaire à tous les publics. Ainsi, au contraire de la structure de production générique qui avait cours durant la grande période des studios et qui favorisait la spécialisation de la production en fonction des marchés cibles, le mode de production actuel oblige que soient restreints au maximum les écarts par rapport à une norme institutionnelle. Dans ce contexte, on comprend que le contrat de spectature propre à l'espace états-unien de la fiction cinématographique soit des plus contraignants, entraînant dans son sillage réducteur une communauté de spectateurs réticents à la différence.

Cette situation permet par ailleurs d'expliquer le statut d'auteur dans l'institution du cinéma états-unien, et bien sûr les conséquences d'un tel statut sur le contrat de spectature proposé par les films. Là où l'écrivain travaille en solitaire, un peu 
comme un artisan, et que seule l'intervention d'un éditeur ou de conseillers littéraires est susceptible de modifier son produit, l'idée même "d'auteur de cinéma" apparaît en réalité davantage comme une abstraction: exécutants plutôt anonymes, souvent formés à la base comme techniciens, ceux-ci aspirent le plus souvent à une reconnaissance qui leur permettra de travailler avec des budgets de plus en plus imposants. En outre, à Hollywood, la structure de production des films est encore aujourd'hui largement déterminée par les genres. Bien sûr, ceux-ci ont changé, ils collent aujourd'hui à une réalité différente; la tâche du réalisateur hollywoodien n'en est pas moins de s'effacer derrière les règles constitutives de ces genres (comédies romantiques, thrillers, drames psychologiques, etc.).

Dans ce contexte ne devient pas "auteur" qui veut; l'accès à ce statut est difficile et passe généralement par une formation différente de celle reçue à Hollywood: par la consécration de certaines instances de légitimation; par la réalisation également d'un film ou deux qui sont unanimement reconnus comme personnels et achevés, malgré des limites importantes dans les moyens de production. Plus importants encore, la forme et le contenu des films dits d'auteurs suivent d'assez près un certain nombre de constantes où se trouvent à la fois repris et reflété ce statut lui-même. On pourrait résumer ce statut de la façon suivante: 1) l'auteur de cinéma états-unien, de par sa formation et ses intérêts, se pose comme un "artiste" ou un "intellectuel " dans un monde de financiers et de techniciens; il est donc essentiellement en position contre-institutionnelle, ou à tout le moins il travaille à donner cette image de lui-même. 2) Ce statut d'artiste lui fait choisir d'emblée de travailler "en marge des genres", ou alors d'adopter à leur égard une attitude de renouveau radical. Il y oppose le plus souvent une forme plus ou moins caractérisée de style, qui devient en quelque sorte sa marque d'auteur. L'accès au statut d'auteur peut aussi passer par une sorte de maîtrise absolue d'un genre en particulier, qu'il contribue à fonder ou à développer (on pense par exemple à Tarantino pour le film de gangsters ultraviolent, à De Palma pour le thriller, etc.). 3) Artiste ou intellectuel en marge de l'institution dominante, l'auteur de cinéma s'installe d'emblée dans un 
"projet esthétique" de défense des intérêts les plus élevés du "cinéma pour le cinéma". Se multiplient alors les déclarations chocs sur l'état de l'industrie (Coppola, Altman), différents types d'attitude de conservation (Woody Allen et Martin Scorsese s'opposant à la coloration des vieux films en noir et blanc), un comportement qu'on pourrait qualifier de "parnassien" dans son esprit. 4) Tout cela se traduit, dans les films euxmêmes comme dans la publicité dont on les entoure, par une "position d'énonciation" où s'affirme et s'affiche "l'autorité" du cinéaste, et la conscience qu'il a de s'adresser à un certain public, essentiellement cinéphilique, qui partage avec lui un ensemble de caractéristiques. Ainsi le meilleur vendeur d'un film d'auteur est bien son appartenance à cette catégorie même, dans une sorte de circularité où on oppose à la logique du marché une logique contre-institutionnelle de l'art pour l'art. Tous ces éléments qui définissent le statut de l'auteur dans le système de production se retrouvent largement reproduits dans leurs œuvres, autour de certaines constantes (stylistique, thématique, générique, etc.). D'où le recours de plus en plus fréquent des auteurs à un contrat de spectature que nous nommons le "contrat de métafiction" où domine une attitude ouvertement réflexive qui vient refléter leur position particulière par rapport à l'institution dominante.

Enfin, peut-être sommes-nous mieux en mesure de comprendre dans ce contexte l'accueil qui est généralement réservé au cinéma étranger aux États-Unis, y compris le cinéma québécois. Il est trop facile de s'en tenir aux lieux communs sur cette question, en proposant, par exemple, que le protectionnisme culturel serait responsable de l'imperméabilité des frontières cinématographiques états-uniennes aux produits étrangers. C'est vers la nature même de l'institution du film de fiction des États-Unis qu'il faut regarder, vers l'extraordinaire prégnance d'un certain contrat de spectature qui lie la sphère de production à la masse des spectateurs états-uniens, et qui crée, entre autres effets pervers, une sorte d'accoutumance dont la conséquence directe semble être l'impossiblité pour ce public d' "honorer" d'autres contrats. 


\section{OUVRAGES CITÉS}

Baudry, Jean-Louis. L'Effet-cinéma. Paris: Albatros, 1978.

Benveniste, Émile. Problèmes de linguistique générale. Paris: Gallimard,1966.

Bordwell, David. Narration in the Fiction Film. Madison: Wisconsin University Press, 1985.

Bordwell, David. «A Case for Cognitivism ». Iris, n 9 (1989), p. 11-40.

Casetti, Francesco. "Pragmatique et théorie du cinéma aujourd'hui ", Hors-cadre, $\mathrm{n}^{\circ} 7$ (1989), p. 93-98.

Casetti, Francesco. D'un regard l'autre. Le film et son spectateur. Lyon: Presses universitaires de Lyon, 1990.

Casetti, Francesco et Roger Odin. « De la paléo à la néo-télévision : approche sémiopragmatique ". Communications, $\mathrm{n}^{\circ} 51$ (1990), p. 9-26.

Corrigan, Timothy. A Cinema Without Walls: Movies and Culture After Viet-nam. New Brunswick, N.J. : Rutgers University Press, 1991.

Dubois, Jacques. L'Institution de la littérature. Bruxelles: Labor / Fernand Nathan, 1986.

Eco, Umberto. Lector in Fabula. Paris: Grasset, 1985.

Jost, François. "La narratologie: point de vue sur l'énonciation ". CinémAction, n" 20 (1988), p. 63-66.

Jost, François. Un monde à notre image: Énonciation, cinéma, télévision. Paris: Méridiens-Klincksieck, 1992.

Melançon, Benoît. "Théorie institutionnelle et littérature québécoise", L'Institution littéraire. Sainte-Foy: Institut québécois de recherche sur la culture-Université Laval (1986), p. 27-42.

Metz, Christian. Le Signifiant imaginaire. Paris: Christian Bourgois, 1984.

Müller, J. E. «Pragmatique historique du film: Nouvelle Vague et conception de l'auteur. La réception d'A bout de souffle aux Pays-Bas ". Cinémas, vol. 2, n“ 2-3 (1992), p. 57-87.

Odin, Roger. «Mise en phase, déphasage et performativité dans le Tempestaire de Jean Epstein ". Communications, n 38 (1983a), p. 213-238.

Odin, Roger. "Pour une sémio-pragmatique du cinéma ". Iris, n 1 (1983b), p. 67-81.

Odin, Roger. "Du spectateur fictionnalisant au nouveau spectateur : approche sémiopragmatique ". Iris, n' 8 (1988), p. 121-139.

Odin, Roger. "La sémio-pragmatique du cinéma: sans crise ni désillusion ". HorsCadre, n 7 (1989), p. 77-92.

Staiger, Janet. "Reception Studies: The Death of the Reader n, dans R.-B. Barton (ed.), The Cinematic Text. New York: AMS Press (1989).

Staiger, Janet. Interpreting Films: Studies in the Historical Reception of American Cinema. Princeton: Princeton University Press, 1992. 